\title{
Enhanced microwave absorption properties of exfoliated graphite/epoxy composites filled with different ceramic particles
}

\author{
Sandeep Kumar Singh ${ }^{1}$, M.J. Akhtar ${ }^{1,2}$ and Kamal K Kar ${ }^{1,3^{*}}$ \\ ${ }^{1}$ Department of Materials Science Programme, Indian Institute of Technology Kanpur, Kanpur- \\ 208016, India \\ ${ }^{2}$ Department of Electrical Engineering, Indian Institute of Technology Kanpur, Kanpur-208016, India \\ ${ }^{3}$ Department of Mechanical Engineering, Indian Institute of Technology Kanpur, Kanpur-208016, \\ India \\ *Email:kamalkk@iitk.ac.in
}

Multi fillers in a composite are an effective way to enhance electromagnetic absorption properties of a microwave absorber [1]. In this proposed work various composite based on the exfoliated graphite (EG) with the varying weight percentages of ceramic materials e.g. $\mathrm{Al}_{2} \mathrm{O}_{3}, \mathrm{BaTiO}_{3}, \mathrm{ZnO}$ and $\mathrm{TiO}_{2}$ were fabricated in epoxy media by mechanical blending. The complex permittivity spectra of the fabricated composite samples were measured in the $\mathrm{X}$ band $(8.2-12.4 \mathrm{GHz})$ frequency region. The reported results demonstrates that the addition of the ceramic particles in the EG/Epoxy composites enhances the permittivity and the dielectric losses significantly. The highest reflection loss (RL) values of different ceramics fillers at their respective matching thickness is shown in Figure 1.

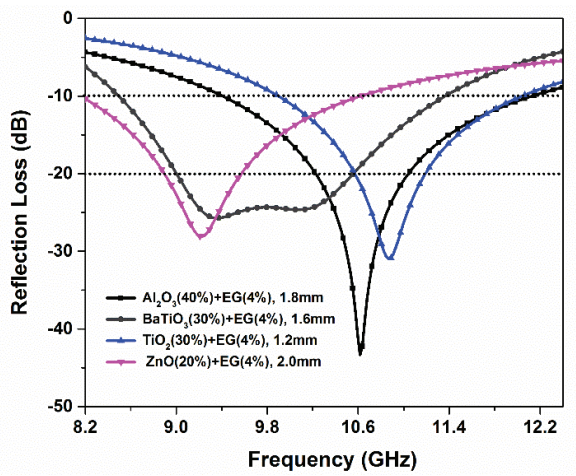

Figure 1: Frequency dependent RL for EG (4\%)/epoxy composites containing various ceramic $\left(\mathrm{Al}_{2} \mathrm{O}_{3}, \mathrm{BaTiO}_{3}, \mathrm{TiO}_{2}\right.$ and $\left.\mathrm{ZnO}\right)$ loadings

The criteria of impedance matching and destructive mechanism phenomena help in achieving the broad band absorption in the composites. The fabricated composite samples were characterized for its morphology analysis using the scanning electron microscopy, which shows the uniform dispersion of the filler materials in the composite. The fabricated samples possessing excellent absorption properties were further tested for its mechanical and thermal properties. Flexural test of the ceramic incorporated composites shows better flexural strength as compared to the EG/epoxy composites as presented in Figure 2. A marked improvement in thermal conductivity of the composites was also observed as compared to the EG/epoxy composite. The findings provide that the significant improvement in the absorption and bandwidth is attributed due to the perfect matching condition and the multiple internal reflections inside the absorber composite [2].

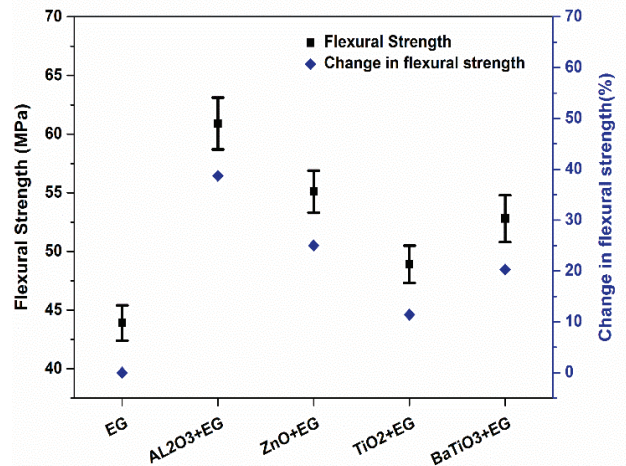

Figure 2: Flexural strength and change in flexural strength (relative to EG/epoxy composites) for EG/ceramic/epoxy composites

\section{References}

1. Y. Qing, X. Wang, Y. Zhou, Z. Huang, F. Luo, W. Zhou, Composites Science and Technology 102, 2014, 161-168

2. C. Liu, Y. Xu, L.Wu, Z. Jiang, B. Shen and Z. Wang, J. Mater. Chem. A, 3, 2015, 10566 